\title{
Introduction of cyber-physical system in robotized press- brake line for metal industry
}

\author{
Beibei Shu ( $₫)$, Gabor Sziebig, Bjørn Solvang \\ UiT, campus Narvik, Lodve Langesgate 2, 8514 Narvik, Norway \\ e-mail: beibei.shu@uit.no
}

\begin{abstract}
Bin picking is a typical work, which is easy to automate up to a given complexity of the work-piece dimensions. In case of casted work-pieces, the dimensions are most of the time not accurate enough for an industrial robot to be able to pick it up without additional sensors/intelligence. In this paper we introduce a cyber-physical system, where all sensors, actuators, machines and industrial robot is connected to a local network, where they share information easily with each other. The novelty of the system that the proposed solution is achieved on the software side, with minimum hardware reconfiguration need. We keep the flexibility of the industrial robot, but extend its' understanding with sensor fusion on a higher decision level, rather than on low robot programming level. The system also allows remote monitoring and supervision of the production plant.
\end{abstract}

Keywords: industrial robot, industry 4.0, cyber-physical system, ROS

\section{Introduction}

In recent years, industry 4.0 has become a very popular topic in industry-related fairs, conferences, or call for public-funded projects. The concept of industry 4.0 was to develop from Internet of Things, which was created by British technology pioneer Kevin Ashton in 1999 (1). Then in April 2013, the Industry 4.0 Working Group present a final report to the German federal government. The report categorizes human industry into four stages, from steam engine stage - first industrial revolution, electrically-powered mass production stage - second industrial revolution and electronics-information stage - third industrial revolution, to cyber-physical based production stage - fourth industrial revolution (2). The fourth stage is the Industry 4.0 which includes cyber-physical system, Internet of things and cloud computing (3). As for now, we are in the industrial revolution but not finish this revolution. Germany is planning to promote the revolution process and Germany is not the only country planning to upgrade their industry. The industry upgrading becomes a trend across the world recently, the representative countries' plan including: Japan - Super Smart Society (4), Norway - Norge 6.0 (5), United States - The Advanced Manufacturing Partnership (6), China - Made in China 2025 (7).

Cyber-physical systems (CPS) are enabling technologies, which bring the virtual and physical worlds together to create a truly networked world in which intelligent objects 
communicate and interact with each other (8). In this paper, we will be introducing a solution for a robotized press-brake line, where all system are connected to the network (a true cyber-physical system) and the progress of the production can be either supervised on-site, in a web browser or in a simulation. This is achieved with the following tools: system based components are connected to the network with the usage of Robot Operating System (introduced in Chapter 2), supervision of the robotized solution is solved by a software solution (called FlexGui 4.0), which is also introduced in Chapter 2. In Chapter 3, the application of the solutions mentioned above will be presented, while Chapter 4 provides the conclusion.

\section{Software based control}

The Robot Operating System (ROS) is an open source, multi-platform robot controlling software, which is a collection of variety tools and libraries (9). ROS is widely used among the researchers, but it is also developed together with industry and is highly promoted between industrial partners, as all results that are achieved in research can be implicitly implemented in industrial version. In ROS, there is a very basic concept Nodes, which are processes that perform computation. For example, one node controls a servo motor, one node control optical sensors and one node control electro-pneumatic valves, see in Fig. 1. Multiple nodes can run in a same ROS device, and multiple ROS devices can connect together. In between all the connected ROS device or inside a ROS device, each node can communicate with each other by passing Messages, which is simply a data structure. Under nodes, there are Topics and Services and a node can contain many topics and services. A topic is like a message bus, see in Fig. 2. You can get messages from a subscribed topic if someone publishes data in it, or send messages to other parties by yourself. A service is like a function, see in Fig. 3. You can call it to run a specific script and wait for a call-back function, but the service can only response one request each time.

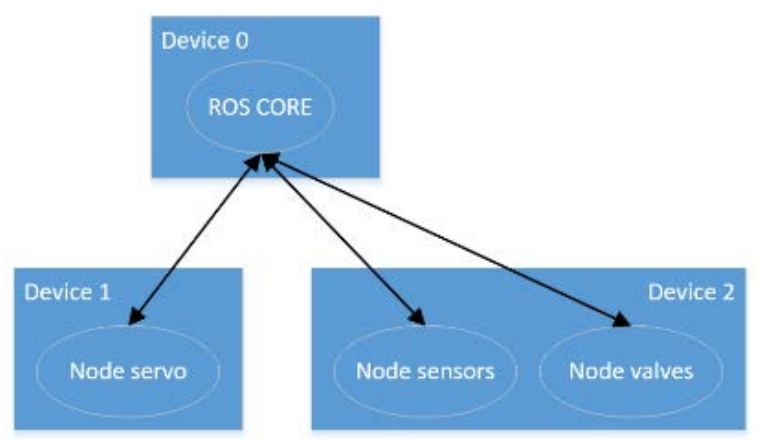

Fig. 1. ROS node 


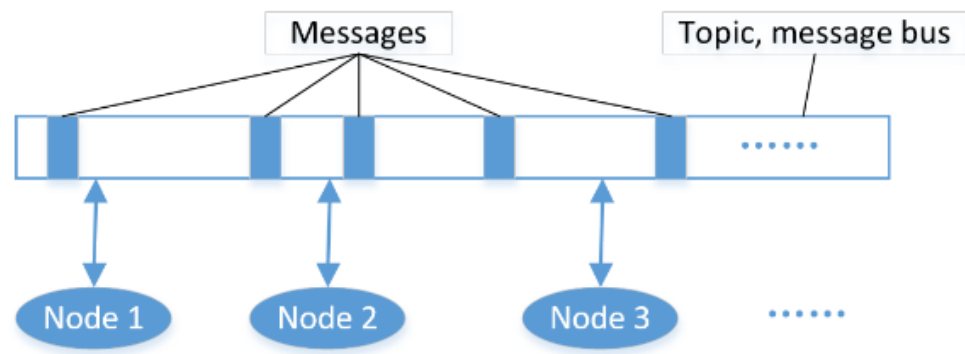

Fig. 2. ROS topic

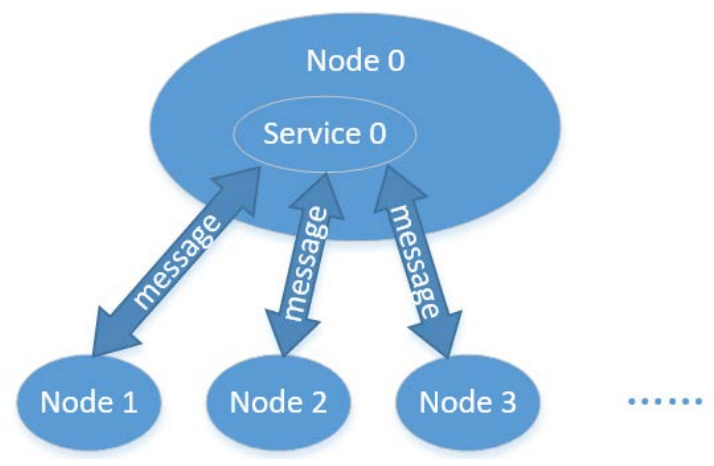

Fig. 3. ROS service

FlexGui 4.0 is an advanced open source user friendly industrial robot controlling interface, which is based upon popular web technologies enabling the possible to run it on almost every modern browser supported device. The user will have exactly the same user experience on each device. FlexGui 4.0 is used to show information and send user inputs to the server, which is the ROS device. Since FlexGui 4.0 is based on ROS, after connected to ROS, FlexGui 4.0 will display all the nodes in the system, and offer a graphic interface for user to read or set variables. For customized variables, user can publish them to topics or send request to services. Based on this mechanism, FlexGui 4.0 is not suit for real-time control functions. While, it is possible for ROS connected to multiple FlexGui 4.0 clients, each FlexGui 4.0 client will synchronize with the server and show the same project, even if it changes only one of them. This makes it is possible for users to develop a project simultaneously (10).

\section{Application}

Gazebo is a well-designed free robot simulator, and has already embedded into ROS. When the Gazebo is running, ROS server will generate a ROS node called "gazebo". Meanwhile, FlexGui 4.0 is connect to ROS server, and it will recognize the node gazebo. User can change a robot-moving project without interfere the process line, and test it virtually in Gazebo. If the project passes the test, user can connect physical robot 
with ROS server, conduct actual test or introduce it into processing line. When the physical robot is running, user can see a simulated robot additionally in Gazebo, Fig. 4.

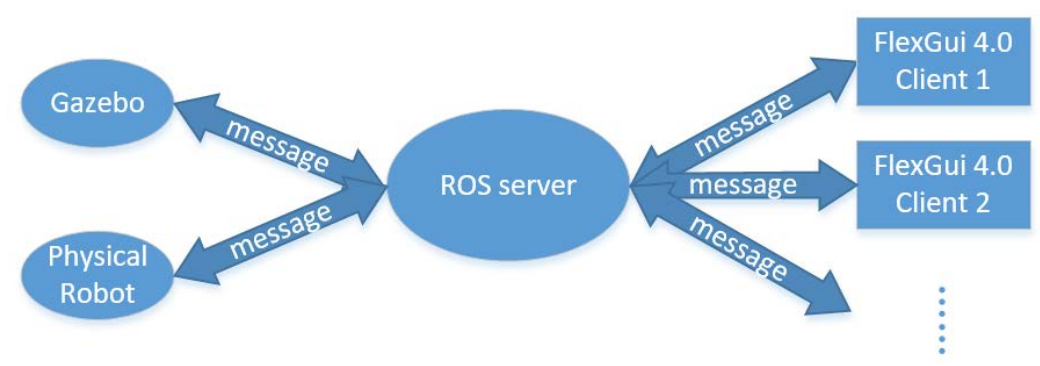

Fig. 4. Connect Gazebo and Physical robot

In Fig. 5, we can see a virtual press-brake line in Gazebo and a robot control interface in FlexGui 4.0. During the simulation or real manufacturing process, the Gazebo will show the robot's activity and FlexGui 4.0 will offer a detail state of each sensor. And for each element on the FlexGui 4.0 interface such as button in this case, we can change the number, the name, the location, the size or the background function of the button. Multiple robot instructions can be integrated in one button. So, the robot will execute a series of actions just after one button clicked.

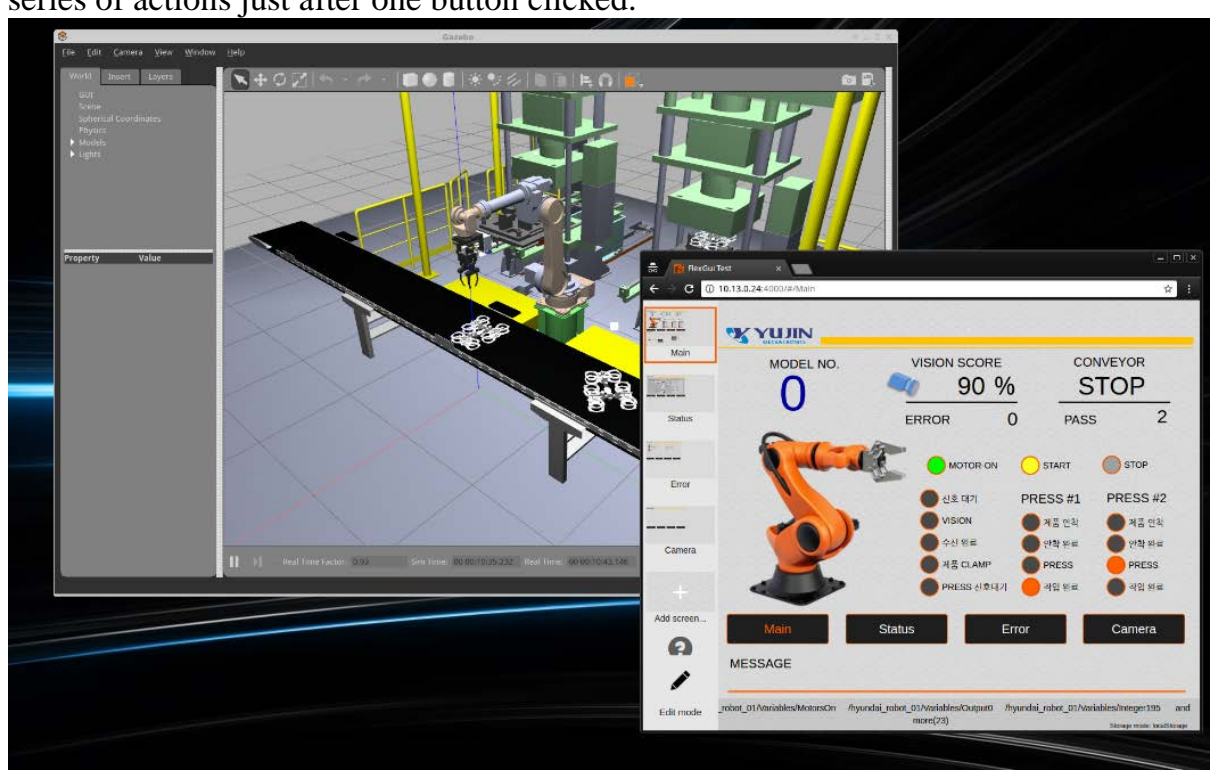

Fig. 5. Gazebo simulation and FlexGui 4.0 interface 


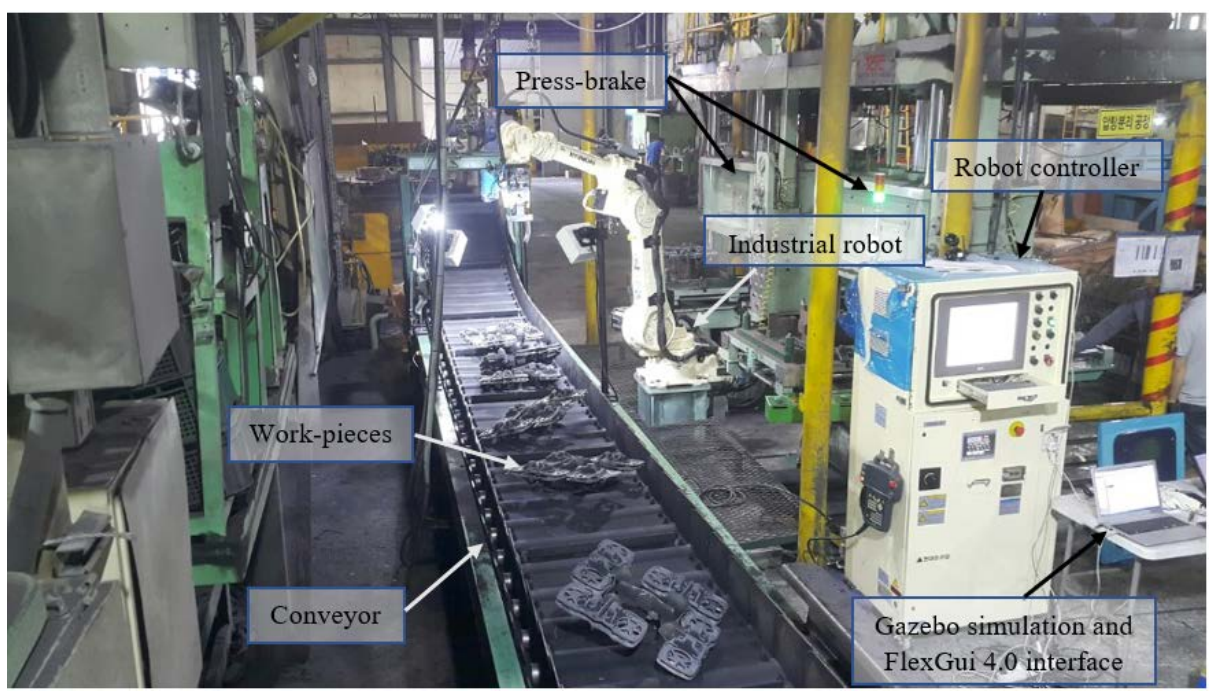

Fig. 6. Industry robot and press-break line in real case

In Fig. 6, that is a real case in physical world using this ROS, FlexGui 4.0 and Gazebo solution. Work-pieces that are coming from the moulding machine, robot picks these up and sets on the press-brake, then two pieces running the production parallel. The conveyor, press-brake, robot controller and all sensors connect to ROS sever, each device will be assigned a node name in ROS. With the help of ROS network, all the objects can easily share information with each other including Gazebo and FlexGui 4.0, achieving Gazebo simulation and FlexGui 4.0 interface control. A demonstration of this communication and supervision for the application can be viewed on the following place: http://t.cn/RSVQGgz

\section{Conclusion}

In this paper, we introduce a cyber-physical system, where all sensors, actuators, machines and industrial robot is connected to a local network - ROS network, where they share information easily with each other by ROS message. The novelty of the system that the proposed solution is achieved on the software side, with minimum hardware reconfiguration need. The only hardware configuration is to connect all the present signal to a ROS supported computer, so ROS can access all the devices in the production line. With the aid of FlexGui 4.0, we keep the flexibility of the industrial robot, but extend its' understanding with sensor fusion on a higher decision level, rather than on low robot programming level. Gazebo synchronously simulate production line with the physical world that allows remote monitoring and supervision of the production plant. 


\section{Acknowledgement}

The research was supported by Korea Institute for Advancement of Technology (Kiat) grant funded by the Korea government (N0001698), Eurostars programme (E-9692) and YUJIN Mechatronics Co., Ltd.

\section{References}

1. Werr P. IoT Evolution World. [Online].; 2015 [cited 2017 May 29. Available from: http://www.iotevolutionworld.com/m2m/articles/401292how-industry-40-the-internet-things-connected.htm.

2. Kagermann H, Wahlster W, Helbig J. Final report of the Industrie 4.0 Working Group. ; 2013.

3. Morgan J. A Simple Explanation Of 'The Internet Of Things'. [Online]; 2014 [cited 2017 May 29. Available from: http://www.forbes.com/sites/jacobmorgan/2014/05/13/simple-explanationinternet-things-that-anyone-can-understand/\#3f87e8a66828.

4. Harayama Y. Society 5.0. [Online].; 2016 [cited 2017 May 29. Available from: http://fpcj.jp/wp/wpcontent/uploads/2016/07/f2d3eec7bf7678840f8adf2ca8000b05.pdf.

5. Qvam W. Norge 6.0. [Online].; 2015 [cited 2017 May 29. Available from: https://khrono.no/sites/default/files/walter_qvam_hjernekraft2015.pdf.

6. The White House Office of the Press Secretary. President Obama Launches Advanced Manufacturing Partnership Steering Committee “2.0”. [Online].; 2013 [cited 2017 May 29. Available from: https://obamawhitehouse.archives.gov/the-press-office/2013/09/26/presidentobama-launches-advanced-manufacturing-partnership-steering-com.

7. Wübbeke J, Meissner M, Zenglein MJ, Ives J, Conrad B. MADE IN CHINA 2025: The making of a high-tech superpower and consequences for industrial countries. ; 2016.

8. Germany Trade \& Invest. INDUSTRIE 4.0 - Smart Manufacturing for the Future. [Online].; 2016 [cited 2017 May 29. Available from: https://www.gtai.de/GTAI/Content/EN/Invest/_SharedDocs/Downloads/GT $\mathrm{AI} /$ Brochures/Industries/industrie4.0-smart-manufacturing-for-the-futureen.pdf? $\mathrm{v}=8$.

9. Thomas D. ROS/Introduction. [Online].; 2014 [cited 2017 May 29. Available from: http://wiki.ros.org/ROS/Introduction.

10. PPMAS. www.ppm.no. [Online].; 2016 [cited 2017 May 29. Available from: https://www.ppm.no/flexgui4-Home/Index/downloads. 